\title{
Carnets
}

Revue électronique d'études françaises de l'APEF

Deuxième série - 6 | 2016

Exotopies de Barthes

\section{Barthes Germinal}

\section{Thomas Vercruysse}

\section{OpenEdition}

\section{Journals}

Édition électronique

URL : http://journals.openedition.org/carnets/748

DOI : $10.4000 /$ carnets. 748

ISSN : 1646-7698

Éditeur

APEF

\section{Référence électronique}

Thomas Vercruysse, «Barthes Germinal », Carnets [En ligne], Deuxième série - 6 | 2016, mis en ligne le

31 janvier 2016, consulté le 19 avril 2019. URL : http://journals.openedition.org/carnets/748 ; DOI :

$10.4000 /$ carnets. 748

Ce document a été généré automatiquement le 19 avril 2019.

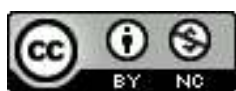

Carnets est mis à disposition selon les termes de la licence Creative Commons - Atribution - Pas d'utilisation commerciale 4.0 International. 


\title{
Barthes Germinal
}

\author{
Thomas Vercruysse
}

\section{NOTE DE L'AUTEUR}

Les références aux œuvres de Barthes renvoient à l'édition des CEuvres complètes réalisée par Éric Marty et publiée au Seuil en 2002. Elles sont présentées suivies du numéro du tome, en chiffres romains, puis des numéros de pages, en chiffres arabes.

Dans Le Degré zéro de l'écriture (1953), Barthes propose une triade célèbre, distinguant « langue », «style » et « écriture » : la langue comme dénominateur commun culturel, le « style », comme " poussée » "d'ordre germinatif » $(O C, I: 177-182)$ et individuel, décrite en termes biologiques, et «l'écriture ", comme responsabilité de l'écrivain engagé dans les formes. Cette triade sera délaissée dans les années 70 : la langue unifiée éclatera dans "la division des langages", le style référera à une réalité culturelle (et non plus individuelle) et l'écriture, réélaborée par les apports du poststructuralisme, deviendra « débordement, emportement du style vers d'autres régions du langage et du sujet, loin d'un code littéraire classé » (Barthes, «L'écriture commence par le style » (1975), OC, IV : 653).

2 Entre les deux triades, la dimension biologique du style semble avoir disparu. Je ne vais pas tenter de sonder les raisons de cet escamotage, et plutôt réfléchir à la façon dont la métaphore du vivant et son champ lexical persiste comme enjeu chez Barthes, et comment cette fidélité discrète voire inconsciente permet de relier Barthes à des préoccupations contemporaines constituant pour nous l'avenir de la poétique. Cet avenir se traduit par une herméneutique générale ne maintenant pas étanches ce que fait l'art et ce que fait la vie, et nous essaierons de montrer que Barthes n'est pas étranger à cette herméneutique, bien qu'il se méfie de l'herméneutique.

3 Reprenons le passage du Degré zéro dans lequel le style est décrit :

La langue est donc en deçà de la Littérature. Le style est presque au-delà (...). Quel que soit son raffinement, le style a toujours quelque chose de brut: il est une forme sans destination, il est le produit d'une poussée non d'une intention, il est comme 
une destination verticale et solitaire de la pensée. (...) Indifférent et transparent à la société, démarche close de la personne, il n'est nullement le produit d'un choix, d'une réflexion sur la Littérature. Il est la part privée du rituel, il s'élève à partir des profondeurs mythiques de l'écrivain, et s'éploie hors de sa responsabilité. Il est la voix décorative d'une chair inconnue et secrète; il fonctionne à la façon d'une Nécessité, comme si, dans cette espèce de poussée florale, le style n'était que le terme d'une métamorphose aveugle et obstinée (...). Le style (...) n'a qu'une dimension verticale, il plonge dans le souvenir clos de la personne, il compose son opacité à partir d'une certaine expérience de la matière (...) (OC, $I: 178)$.

Si on prend la métaphore du style comme " poussée » «d'ordre germinatif » au sérieux, on peut dire que Barthes se trouve à un point de bifurcation philosophique. D'un côté, on trouve l'esthétique de Kant, qui inclut les productions artistiques dans le paradigme englobant des productions de la nature qui constitueraient le modèle indépassable de l'art, de l'autre, celle de Hegel, qui les disjoint, car les productions humaines seraient animées, selon lui, par la liberté, ce qui leur conférerait beaucoup plus de noblesse que les créations naturelles. Mais depuis, le philosophe Jacques Dewitte a, d'une certaine manière, dépassé cette opposition en nous peignant la liberté qui est celle de la vie par le biais de l'autoprésentation, [une autoprésentation «qui n'est pas le fait du hasard » et possède un « caractère intentionnel »]: «C'est ce que Portmann appelle Selbstdarstellung, l'autoprésentation ou la manifestation de soi, une dimension qui est présente à la fois chez l'homme, dans les phénomènes sociaux comme le rite ou la monumentalité, et dans la vie extra-humaine (et que Buytendijk appelait déjà "valeur démonstrative") (Dewitte, $2010: 12$ ).

Barthes parlait, dans «Qu'est-ce que l'écriture » du style comme de la "part privée du rituel » (OCI : 177-182), "forme sans destination », "produit d'une poussée, non d'une intention » (ibid.). Quelle place alors pour la part publique du rituel ? La clé nous est peutêtre donnée dans la Préface aux Essais critiques, publiée en 1964 : « (...) l'auteur ne produit jamais que des présomptions de sens, des formes, si l'on veut, et c'est le monde qui les remplit » (OC, II : 273). Apparemment, nous ne sommes plus dans la première triade, qui n'est pas convoquée: on pourrait bien imaginer que la langue est présupposée, que le style est en amont et que l'écriture informe le style en l'engageant dans le circuit social des valeurs. Cependant, quelque chose de nouveau est apparu, absent de la première triade : la dimension herméneutique assumée par le monde qui sature les sens et les formes offerts par l'écrivain. La réception est prise en compte, à côté de la production, et la complète. Plus loin dans la Préface intervient la métaphore décisive du thème et des variations, métaphore musicale dont j'aimerais montrer qu'elle n'est pas sans lien avec la métaphore biologique :

L'écrivain est un expérimentateur public : il varie ce qu'il recommence; obstiné et fidèle, il ne connaît qu'un art : celui du thème et des variations. Aux variations, les combats, les valeurs, les idéologies, le temps, l'avidité de vivre, de connaître, de participer, de parler, bref les contenus ; mais au thème l'obstination des formes, la grande fonction signifiante de l'imaginaire, c'est-à-dire l'intelligence même du monde. Seulement, à l'opposé de ce qui se passe en musique, chacune des variations de l'écrivain est prise elle-même pour un thème solide, dont le sens serait immédiat et définitif (ibid. : 274-275).

6 Il ne faudrait pas lire cette distinction thème - variations d'après la dichotomie fondforme. Pas non plus d'après le schéma hylémorphique d'Aristote d'après lequel la matière aspire à une forme posée au préalable. Barthes énonce ainsi plus loin : «écrire, c'est ou bien projeter ou bien terminer, mais jamais "exprimer"; entre le commencement et la 
fin, il manque un maillon (...) celui de l'œuvre elle-même ; on écrit peut-être moins pour matérialiser une idée que pour épuiser une tâche qui porte en elle son propre bonheur » ( ibid. : 257).

7 Je crois qu'on peut davantage lire le rapport entre l'écrivain et l'idée d'après le concept de modulation, désignant l'interaction entre force et matériaux, qu'on trouve développé dans la thèse de Simondon dont une partie, L'individu et sa genèse physico-biologique paraît la même année, en 1964. Ce livre sera chroniqué de manière enthousiaste par Deleuze. Simondon n'est pas cité dans la biographie de Tiphaine Samoyault (Samoyault, 2015). Je me livre donc ici à un rapprochement de type analogique, non historique (ce sera, par défaut, notre manière de ne pas céder à ce que Barthes appelait le «mythe de la filiation »). Le concept de modulation intervient chez Simondon à la fois au niveau non organique, mais aussi au niveau du vivant non humain puis humain pour expliquer l'individuation.

8 Une source en revanche directe pour Barthes, c'est Valéry, que Barthes cite régulièrement dans ses œuvres et qui apparaît en filigrane dans la suite du texte : « Il y a une sorte de vocation de l'écriture à la liquidation; et bien que le monde lui renvoie toujours son œuvre comme un objet immobile, muni une fois pour toutes d'un sens stable, l'écrivain lui-même ne peut la vivre comme une fondation, mais plutôt comme un abandon nécessaire » $(O C, I I: 275$.) On croirait lire ici Valéry, qui considérait qu'un poème n'était jamais achevé mais toujours abandonné, abandonné comme une araignée laisse un cocon ou un serpent sa mue (Morimoto, 2009). Or un des modèles de Valéry est Goethe, et, dans le discours qu'il lui consacre, Valéry propose justement un parallèle entre la maîtrise de la forme linguistique de nature poétique et la forme naturelle modelée par la plante: «[ ...] dans le poète ou dans la plante, c'est le même principe naturel: tous les êtres ont une aptitude à s'accommoder, et cette aptitude variable mesure leur aptitude à vivre, c'est-à-dire à demeurer ce qu'ils sont, en possédant plus d'une manière d'être ce qu'ils sont» (Valéry, 1957: 538). Valéry rapproche ici le conatus, concept de Spinoza, première influence de Goethe, par lequel le poète et la plante persévèrent dans leur être ("demeurer ce qu'ils sont») avec le potentiel transformateur (" possédant plus d'une manière d'être ce qu'ils sont »). Le lieu de leur articulation est le kairos, la circonstance (dont Barthes fait grand cas dans le cours sur Le Neutre), circonstance qui détermine les variations de l'être dont le devenir est une dimension, comme l'ont montré les philosophies de Simondon mais aussi de Nietzsche (qui considérait Spinoza comme un frère et que Barthes connaissait aussi via Nietzsche et la philosophie de Deleuze [Deleuze, 1962]).

9 On a retrouvé ici l'idée du thème et des variations, à partir, chez Goethe, de l'archétype de la plante originaire, l'Urpflanze, qui diffère en s'incarnant dans des variations. Quel rapport avec Barthes? Barthes appréciait Goethe, T. Samoyault le souligne, mais en mentionnant Werther (présent dans les Fragments du discours amoureux) non les écrits sur la Morphologie des plantes. Mais il hérite de Goethe cette idée du thème et des variations par un autre biais, celui des formalistes russes (qu'il cite plus loin dans la préface) en particulier de Propp et sa Morphologie du conte que Lévi-Strauss l'a incité à lire lors de leur fameuse rencontre de 1960, où Barthes lui demande de diriger sa thèse. Or la folkloristique de Propp correspond à une application de la morphologie goethéenne des sciences de la nature aux sciences de la culture, fidèle au geste des formalistes russes dépassant la division diltheyienne entre Naturwissenschaften et Geisteswissenschaften. Barthes confie avoir lu la Morphologie du conte, en anglais (Samoyault, 2015 :354). On sait 
que Lévi-Strauss refusera de diriger la thèse de Barthes et $\mathrm{T}$. Samoyault est revenue sur les raisons de ce refus. Elle pointe notamment un désaccord scientifique : le statut de la structure. Parmi les différences évoquées, on relèvera celle-ci : "Différence dans le projet : dévoiler chez Barthes; restituer les conditions d'exercice de la pensée symbolique chez Lévi-Strauss » (Samoyault, 2015 : 356).

On va essayer ici de déplacer cette différence. Si le dévoilement, propre à l'activité herméneutique, n'est pas conciliable avec la méthode structuraliste selon Lévi-Strauss (c'est la position qu'il défend face à Ricœur lors du débat organisé par la revue Esprit en 1963 [Dosse, 2012 :280]), Barthes, pour défendre « La Critique » dans Critique et vérité, en 1966, met l'accent, Claude Coste le souligne, sur «l'ensemble des contraintes qui s'imposent au critique et rendent sa parole recevable : prise en compte de la totalité des phénomènes, logique de la contrainte symbolique (Coste, 2010 : 179) [Coste, $2010: 159]$ ) ». Cette logique de la contrainte symbolique est propre au structuralisme, dont Deleuze écrivait que son ambition n'était pas d'ordre quantitatif mais topologique et relationnel (Deleuze, 1979). Or la topologie, contrairement aux mathématiques de la quantité, fait droit aux processus de formation-déformation, ceux qu'observe D'Arcy Thompson dans l'évolution des formes animales et relatées dans On Growth and Form (D'Arcy Thompson, 2009). Lévi-Strauss affirmait que son idée de la transformation structurale lui serait venue principalement de la biologie de d'Arcy (Lévi-Strauss, 1988: 158-159). D'Arcy disait luimême s'être inspiré des recherches de Dürer sur l'anamorphose. Et, précisément, Barthes joue la carte de l'anamorphose contre l'herméneutique dans Critique et vérité pour définir l'activité critique : «Il s'agit en somme d'une sorte d'anamorphose, étant bien entendu (...) que l'anamorphose (...) est une transformation surveillée, soumise à des contraintes optiques" (OC, II : 792-799). En cela, l'anamorphose est analogue à une opération de modulation chez Simondon. Quand Barthes rejette l'herméneutique comme recherche du sens caché d'un texte, il en expose les raisons : «Le rapport de la critique à l'œuvre est celui d'un sens à une forme. Le critique ne peut prétendre "traduire" l'œuvre, notamment en plus clair, car il n'y a rien de plus clair que l'œuvre. Ce qu'il peut c'est "engendrer" un certain sens en le dérivant d'une forme qui est l'œuvre. » (ibid.)

11 En fait, Barthes reste tributaire d'une conception de l'herméneutique gagée sur la clarté, issue de Parménide, propre à une sémiotique logico-grammaticale qui lui a été transmise par Hjelmslev. Cette tradition est animée par l'idée que le sens littéral est voilé et serait à restituer. Cette problématique de la clarté dépend « d'une théorie de la révélation au sens large » qui procéderait ou bien « de la bienveillance divine répandue sur l'écriture » ou bien, depuis les Lumières, bien nommées, la clarté serait le produit d'une représentation rationnelle de l'ordre naturel, l'obscurité ne représenterait qu'une difficulté provisoire procédant du préjugé et appelée à être levée par la connaissance (Rastier, 2001 :113).

Or Barthes aurait pu se reconnaître dans une herméneutique de la difficulté, issue d'Héraclite. On a affaire ici à un autre type de révélation, qui n'est pas fondé sur la vision immédiate, comme dans la problématique de la clarté, mais sur l'ouïe, ou plutôt l'entente. La pythie ou la Sybille s'expriment de manière oblique, le sens est, d'une certaine manière, anamorphosé : il n'est pas à restituer, mais à constituer : dans cette tradition, on peut ranger Heinz Wismann, Jean-Michel Salanskis, François Rastier et Stanley Fish. Le sens n'est pas immanent au texte mais à l'interprétation. On peut alors reprendre la citation qu'on a mentionnée plus haut: "le sens d'une œuvre ne peut se faire seul; l'auteur ne produit jamais que des présomptions de sens, des formes si l'on veut, et c'est le monde qui les remplit ». 
Ce n'est pas seulement le monde dans sa socialité qui remplit le sens. Barthes rappelle dans Le Neutre que « le langage est pour l'homme un véritable milieu biologique, ce dans quoi il vit et par quoi il vit, ce qui l'entoure» (Barthes, 2002: 122-123) Barthes, implicitement, nous encourage à placer l'herméneutique textuelle, celle de la difficulté, en relation de continuité avec une herméneutique générale qui peut informer la question du vivant. Le sens dans la lecture participe des échanges gazeux et plus largement énergétiques avec le milieu, avec le système de circonstances. Le lecteur, comme l'auteur, sont inspirés, pour ce qu'ils respirent leur système de circonstances. La création dans la lecture comme dans l'écriture relève bien d'une métabolisation c'est pourquoi l'herméneutique «intégrée » de Marielle Macé, qu'on peut ranger parmi les spécialistes et les disciples de Barthes (Macé, Gefen, 2003) ne se sépare pas d'une philosophie de la vie qui lui a été transmise, pour une bonne part, par Simondon, comme j'ai tenté de le montrer ailleurs (Vercruysse, 2015). De ce point de vue, Barthes et Simondon sont, dans son œuvre, présents et intériorisés, comme le monde, social et biologique, est pour le lecteur et l'auteur, tout à la fois présent et intériorisé. Le monde est devant nous et en nous, comme Barthes.

\section{BIBLIOGRAPHIE}

BARTHES, Roland (2002). Le Neutre, cours au collège de France, 1977-1978. Paris : Seuil.

BARTHES, Roland (1953). « Le degré zéro de l'écriture » (2002), CEuvres complètes I. Paris : Seuil.

BARTHES, Roland (1953). « Qu'est-ce que l'Ecriture ?». (2002), Euvres complètes I. Paris : Seuil.

BARTHES, Roland (1964). « Préface, Essais critiques ». (2002), Euvres complètes II. Paris : Seuil.

BARTHES, Roland (1975). Roland Barthes par Roland Barthes. (2002), Euvres complètes IV. Paris : Seuil.

coste, Claude (2010). Barthes. Paris : Seuil, « Bibliothèque ».

D'ARCY, Thompson (2009). Forme et croissance. trad. française D. Teyssié, Paris : Seuil.

DELEUZE, Gilles (1962). Nietzsche et la philosophie. Paris : Puf.

DELEUZE, Gilles [1973] (1979). « À quoi reconnaît-on le structuralisme », in François Châtelet (dir.), Histoire de la philosophie. La Philosophie du $\mathrm{Xx}^{e}$ siècle, Paris : Hachette, t. 4.

DEWITTE, Jacques (2010). La Manifestation de soi - éléments d'une critique philosophique de l'utilitarisme. Paris : La Découverte.

DOSSE, François [1991] (2012). Histoire du structuralisme, Le champ du signe. Paris : La Découverte, "Poche ", t. 1.

LÉVI-STRAUSS, Claude, Éribon Didier (1988). De près et de loin. Paris : Odile Jacob.

MACÉ, Marielle, Alexandre Gefen (dir.) (2003). Barthes au lieu du roman. Paris : Desjonquères.

MORIMOTO, Atsuo (2009). L'Imaginaire et la genèse du sujet - Paul Valéry, de la psychologie à la poḯtique. Paris : Minard. 
RASTIER, François (2001). Arts et sciences du texte. Paris : Puf.

SAMOYAULT, Tiphaine (2015). Roland Barthes. Paris : Seuil.

VALÉRY, Paul (1957). « Discours sur Goethe », in Euuvres complètes, I. éd. Jean Hytier, Paris :

Gallimard, « Pléiade ».

VERCRUYSSE, Thomas (2015). « Façons de lire, manières de devenir : la lecture comme occasion

d'une éthopoétique »

http://www.epistemocritique.org/spip.php?article403\&lang=fr

\section{RÉSUMÉS}

Cette étude cherche à analyser comment la métaphore du vivant livre des enjeux cruciaux de la poétique de Barthes, le rapprochant des préoccupations les plus actuelles en herméneutique, qu'on songe à Stanley Fish, Marielle Macé mais aussi Heinz Wismann. L'avenir de la poétique consisterait alors à ne pas séparer l'art du vivant.

This paper tries and examines how the metaphor of the living gives high stakes of Barthes' poetics, putting him together with the most current considerations in hermeneutics: one can think of Stanley Fish, Marielle Macé but also of Heinz Wismann. The future of poetics would consist of not separating art from the living.

\section{INDEX}

Mots-clés : poétique, vivant, style, herméneutique, forme

Keywords : poetics, living, hermeneutics, form

\section{AUTEUR}

\section{THOMAS VERCRUYSSE}

Université de Neuchâtel

thomas.vercruysse@free.fr 